\title{
On the b-chromatic number of $T_{n}$ and $M\left(\mathrm{~T}_{\mathrm{n}}\right)$
}

\author{
Nadeem Ansari ${ }^{1}$, R S Chandel ${ }^{2}$, Rizwana Jamal ${ }^{3}$ \\ ${ }^{1}$ Department of Mathematics, IES, IPS Academy, Indore-452001, India. \\ ${ }^{2}$ Department of Mathematics, Govt. Geetanjali Girls College, Bhopal-462001, India. \\ ${ }^{3}$ Department of Mathematics, Saifia Science College, Bhopal-462001, India.
}

\begin{abstract}
Theset of vertices $V(G)$ and set of edges $E(G)$ forms a mathematical structure, which is called graph $G$. A $b$-coloring of a graph $G=(V, E)$ is a coloring $f_{G}: V(G) \rightarrow C$ such that, for each $c$ in $C$, there exists some vertex vin $V(G)$ which is colored by $c$ and $f_{G}\left(N_{G}(v)\right)=C \backslash\{c\}$.In this paper, we give the exact value for the $b$-chromatic number of triangular snake graph and middle graph of triangular snake graph, which is denoted by $T_{n}$ and $M\left(\mathrm{~T}_{\mathrm{n}}\right)$ respectively.
\end{abstract}

Keywords:b-coloring, Triangle snake graph, Middle graph.

Corresponding Author: Nadeem Ansari

\section{Introduction}

A graph $G$ is a pair of sets $V(G)$ and $E(G)$, where $V(G)$ and $E(G)$ are referred as the vertex set and edges set of a graph $G$ respectively. We consider finite undirected graphs without loops or multiple edges. Definitions not given here may be found in [3]. Proper vertex coloring of a graph $G$ is an assignment of colors $C$ to the vertices of the graph, in such a way that adjacent vertices is colored differently. If $|C|=k$, we say $C$ is $k$-coloring.Equivalently a proper coloring is a partition of the vertex set $V$ into independent sets $V_{1}, V_{2} \ldots V_{k}$. The sets $V_{i}$ are called color classes. The chromatic number of $G$ is the minimum integer $\chi(G)$ such that $G$ has a proper coloring with $\chi(G)$ colors. A graph $G$ is $k$-chromatic if $\chi(G)=k$. The concept of chromatic number has been extended to almost all types of graphcolorings.

A $b$-coloring of a graph $G$ with $k$ colors is a proper coloring such that each color class contains a vertex with its neighbors in all other $\mathrm{k}-1$ color classes. A vertex of color $i$ that has all other colors in its neighborhood is called color $i$ dominating vertex. The b-chromatic number of a graph $G$, denoted by $\varphi(G)$, is the maximum $k$ for which $G$ has a $b$-coloring by $k$ colors.

This concept was introduced in 1999 by Irving andManlove [7], by considering proper colorings that are minimal with respect to a partial order defined on the set of all the partitions of vertices of G.It is easy to notice that $\varphi(G) \leq m(G)$, where $m(G)$ is the largestinteger $m$ such that $G$ has $m$ vertices of degree at least $m-1$. For a graph $G$ and an integer $k$, the problem of deciding whether $\varphi(G) \geq k$ is $N P$-complete[7].It is an NP-hard to determining the $b$-chromatic number in general but they proved that it is polynomial for trees [7]. The complexity issues of $b-$ colorings were further studied in $[4,9]$.

The discussion on the b-chromatic number of some powergraphs is carried out by Effantin and Kheddouci [5, 6]. The $b$-coloring of regular graphs is studied by Blidia et al.[2], 
while the $b$-coloring of regular graphs without 4 cycle is studied by Shaebani [10].Ramin Javadi and Behnaz Omoomi, studied the $b$-chromatic number for knesar graph [8] and proved that $K(n, 2)$ is $b$-continuous when $n \geq 17$. The $b$-chromatic number of some special graph classes has been discussed in $[1,11]$. In this paper, we present exact values for the b-chromatic number of triangular snake graph andmiddle graph of triangular snake graph.

\section{Preliminaries}

The Triangular cactusis a connected graph all of whose blocks are triangles. It is obtained from a path $P=v_{1}, v_{2}, v_{3}, \ldots, v_{n+1}$ by joining $v_{i}$ and $v_{i+1}$ to a new vertex $u_{i} ; 1 \leq i \leq n$. A triangular snake has $2 n+1$ vertices and $3 n$ edges, where $n$ is the number of blocks in the triangular snake. It is denoted by $T_{n}$. (See Figure 1 )

Let $G$ be a graph with vertex set $V(G)$ and edge set $E(G)$. The middle graphof $G$, denoted by $M(G)$ is defined as follows. The vertex set of $M(G)$ is $V(G) \cup E(G)$. Two vertices $x$; $y$ in the vertex set of $M(G)$ are adjacent in $M(G)$ in case one thefollowing holds: (i) $x$; $y$ are in $\mathrm{E}(\mathrm{G})$ and $x$; yare adjacent in $G$. (ii) $x$ is in $V(G), y$ is in $E(G)$, and $x ; y$ are incident in $G$.

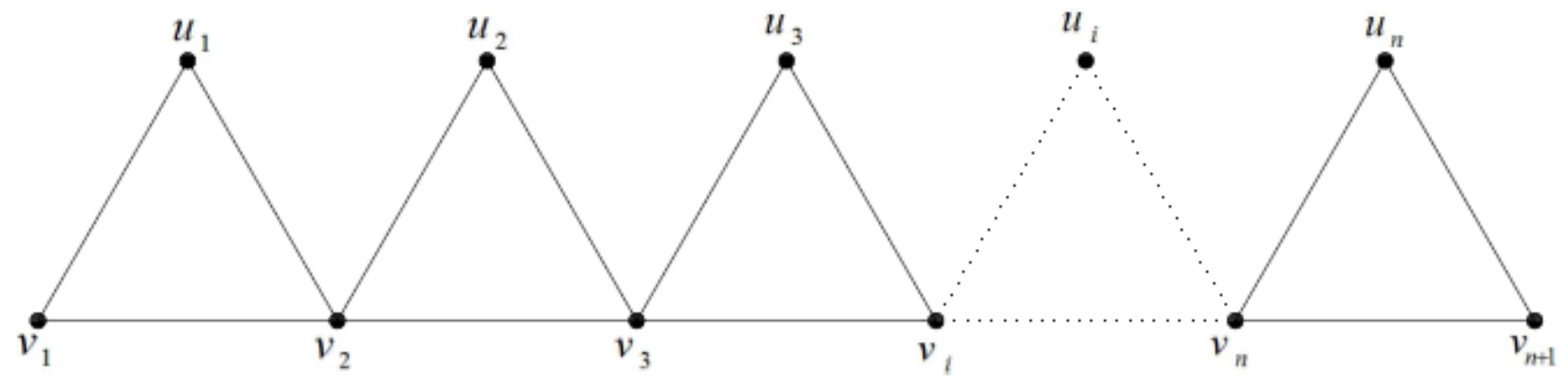

Fig 1: Triangular snake graph $T_{n}$

\section{3. b-chromatic number on $T_{n}$ and $M\left(\mathrm{~T}_{\mathrm{n}}\right)$}

Theorem 3.1:If $n \geq 6$, then the b-chromatic number of triangular snake graph is $\varphi\left\{T_{n}\right\}=5$.

Proof:Let $T_{n}$ be the triangular snake graph with $2 n+1$ vertices and $3 n$ edges. Let $\left\{v_{1}, v_{2}, \ldots \ldots, v_{n+1}, u_{1}, u_{2}, u_{3}, \ldots ., u_{n}\right\}$ be the vertices of the triangular snake $T_{n} . \operatorname{SoV}\left(Y_{n}\right)=$ $\left\{v_{1}, v_{2}, v_{3}, \ldots, v_{n+1}\right\} \cup\left\{u_{1}, u_{2}, u_{3}, \ldots, u_{n}\right\}$.(See Figure 1)

For $n \geq 6, T_{n}$ has at least five vertices of degree 4 . Thus $\varphi\left(T_{n}\right) \leq m\left(T_{n}\right)=5$. By assigning proper coloring to the vertices $\operatorname{asc}\left(v_{1}\right)=5, c\left(v_{i+1}\right)=i$; for $1 \leq i \leq 5$ and $c\left(v_{7}\right)=1$. Now assign $c\left(u_{i}\right)=i+2$; for $1 \leq i \leq 3$ and $c\left(u_{i+3}\right)=i$; for $1 \leq i \leq 3$. For remaining vertices of $T_{n}$, assign $c\left(v_{i}\right)=3 ; 8 \leq i \leq n+1$ and $c\left(u_{i}\right)=2 ; 7 \leq i \leq n$, this implies that $\varphi\left(T_{n}\right) \geq$ 5.Hence the theorem.

Theorem 3.2: If $M\left(\mathrm{~T}_{\mathrm{n}}\right)$ is middle graph of triangular snakegraphthen

$$
\varphi\left\{M\left(\mathrm{~T}_{\mathrm{n}}\right)\right\}=\left\{\begin{array}{l}
7 ; \text { for } 4 \leq n \leq 10 \\
9 ; \quad \text { for } n \geq 11
\end{array}\right.
$$


Proof:Let $T_{n}$ be the triangular snake graph with $2 n+1$ vertices and $3 n$ edges. Let $\left\{v_{1}, v_{2}, \ldots \ldots, v_{n+1}, u_{1}, u_{2}, u_{3}, \ldots \ldots, u_{n}\right\}$ be the vertices of the triangular snake $T_{n}$.Now by definition of middle graph, each edge of graph is subdivided by a new vertex. Therefore assume that each edge $\left(v_{i}, v_{i+1}\right)$ and the line joining $v_{i}$ and $v_{i+1}$ to a vertex $u_{i}, i=1,2,3, \ldots, n$ are subdivided by the vertices $e_{i}, e_{i}^{\prime}$, and $e_{i}^{\prime \prime}, i=1,2,3, \ldots, n$ respectively.(See Figure 2)

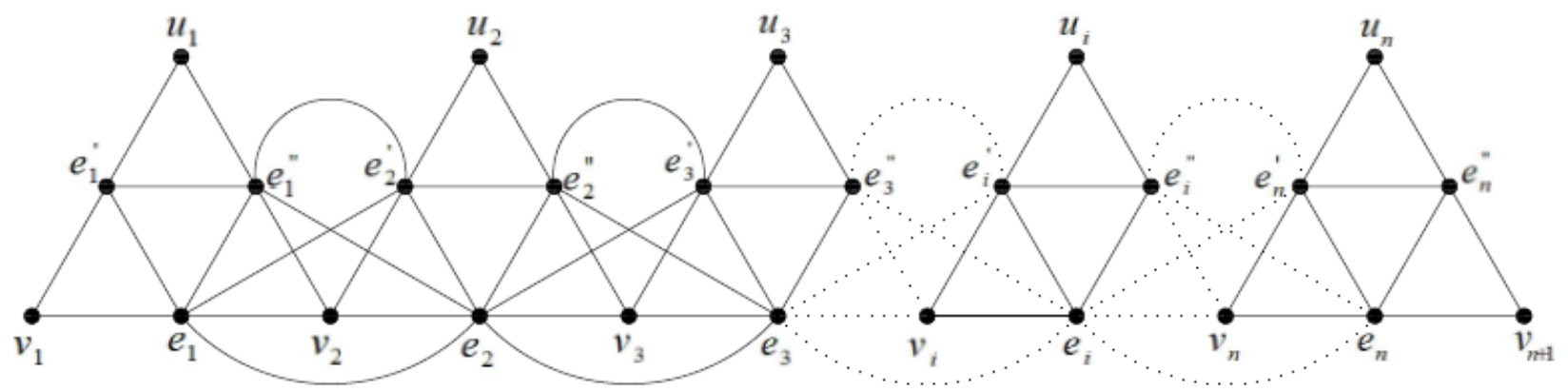

Fig 2: Middle graph of Triangular snake graph $M\left(\mathrm{~T}_{\mathrm{n}}\right)$

For $4 \leq n \leq 10, M\left(\mathrm{~T}_{\mathrm{n}}\right)$ has at least 7 vertices of degree 6 , Then $\varphi\left\{M\left(\mathrm{~T}_{\mathrm{n}}\right)\right\} \leq$ $m\left\{M\left(T_{n}\right)\right\}=7$, for $4 \leq n \leq 10$.Now since the 7-coloring $c: V\left(M\left(Y_{n}\right)\right) \rightarrow\{1,2,3, \ldots, 7\}$ is defined by $\quad c\left(e_{i}\right)=i ; 1 \leq i \leq 4, \quad c\left(e_{1}^{\prime}\right)=6, c\left(e_{2}^{\prime}\right)=7, c\left(e_{3}^{\prime}\right)=1, c\left(e_{4}^{\prime}\right)=6, c\left(e_{i}^{\prime \prime}\right)=i+4 ; 1 \leq i \leq$ $3, c\left(e_{4}^{\prime \prime}\right)=1, c\left(v_{i}\right)=i+2 ; 1 \leq i \leq 3, c\left(v_{4}\right)=5, c\left(v_{5}\right)=2, c\left(u_{1}\right)=c\left(u_{2}\right)=3, c\left(u_{3}\right)=$ $2, c\left(u_{4}\right)=2$. So $c$ is $b$-coloring. Thus for $4 \leq n \leq 10, \varphi\left\{M\left(\mathrm{~T}_{\mathrm{n}}\right)\right\} \geq 7$. Therefore $\varphi\left\{M\left(\mathrm{~T}_{\mathrm{n}}\right)\right\}=$ $7,4 \leq n \leq 10$.(See Figure 3)

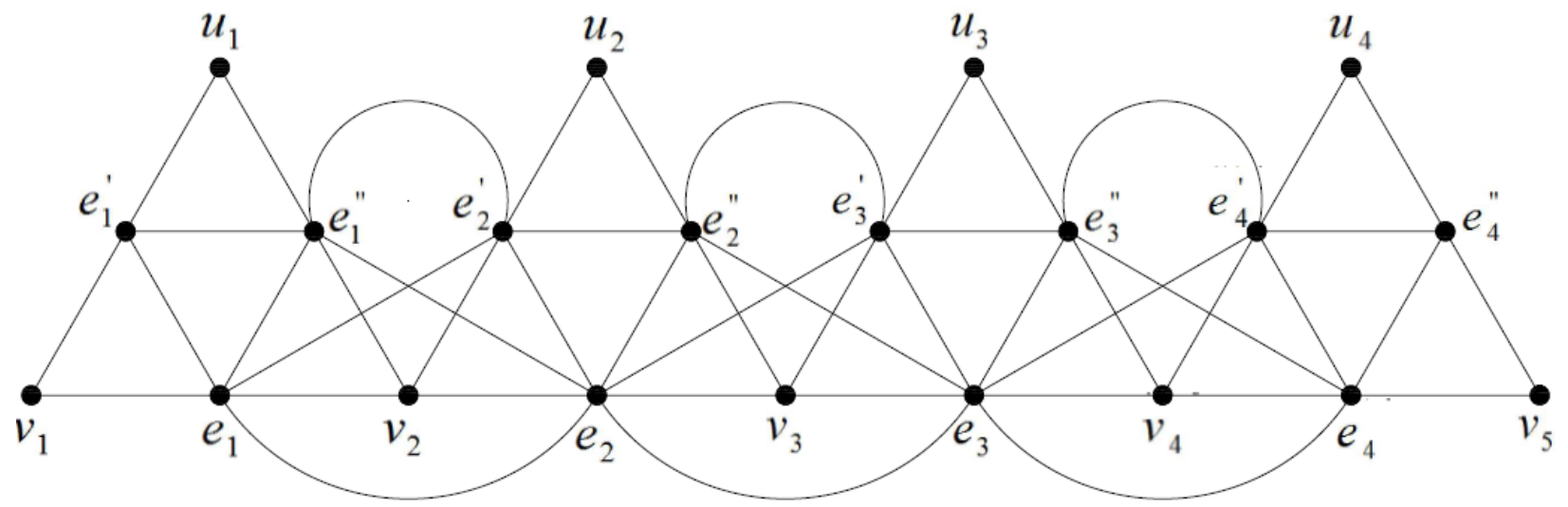

Fig 3: Example $M\left(\mathrm{~T}_{4}\right)$

For $n \geq 11$,consider color class $C=\{1,2,3,4,5,6,7,8,9\}$. Assign b-coloring to $M\left(\mathrm{~T}_{\mathrm{n}}\right)$ as follows.c $\left(e_{1}\right)=9, c\left(e_{i+1}\right)=c\left(u_{i}\right)=i, 1 \leq i \leq 9, c\left(e_{11}\right)=1, \quad c\left(u_{10}\right)=1, c\left(u_{11}\right)=2$, $c\left(v_{i}\right)=3+i ; \quad 1 \leq i \leq 6, \quad c\left(v_{6+i}\right)=i ; \quad 1 \leq i \leq 6$. Now assign $c\left(e_{i}^{\prime}\right)=i+1 ; \quad 1 \leq i \leq 8$, $c\left(e_{8+i}^{\prime}\right)=i ; \quad 1 \leq i \leq 3, c\left(e_{i}^{\prime \prime}\right)=6+i ; 1 \leq i \leq 3, \quad c\left(e_{3+i}^{\prime \prime}\right)=i ; 1 \leq i \leq 8$.For $\quad$ remaining vertices of $M\left(\mathrm{~T}_{\mathrm{n}}\right) ; n \geq 11$, we can apply any proper coloring. Therefore for $n \geq 11$, $\varphi\left\{M\left(\mathrm{~T}_{\mathrm{n}}\right)\right\} \geq 9$. Now for $n \geq 11, M\left(T_{n}\right)$ has at least nine vertices of degree 8.It follows that $\varphi\left\{M\left(\mathrm{~T}_{\mathrm{n}}\right)\right\} \leq m\left\{M\left(T_{n}\right)\right\}=9$, for $n \geq 11$.Thus $\varphi\left\{M\left(\mathrm{~T}_{\mathrm{n}}\right)\right\}=9 ; n \geq 11$.(See Figure 4) 


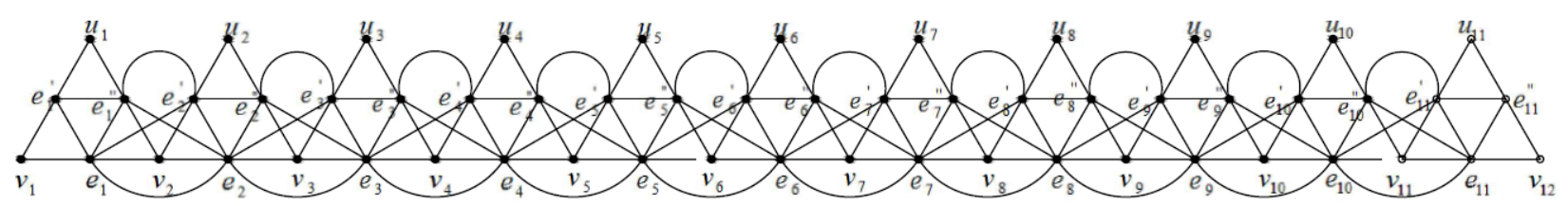

Fig 4: Example $M\left(\mathrm{~T}_{11}\right)$

\section{Conclusion}

b-coloring play important role in clustering, automatic reading system and distributed system. We have investigated b-chromatic number of triangular snake graph and middle graph of triangular snake graph. The investigation of similar results for different graphs as well in the context of various graph coloring problems is an open area of research.

\section{References}

[1] N. Ansari, R. S. Chandel, R. Jamal, THE b-CHROMATIC NUMBER OF HELM GRAPH, International Journal, 15: 8(7), 2017.

[2] M. Blidia, F. Maffray and Z. Zemir, On b-colorings in regular graphs, Discrete Applied Mathematics, Vol. 157, 1787-1793, 2009.

[3] J. A. Bondy and U. S. R. Murty, Graph Theory, Springer, Berlin, 2008.

[4]S. Corteel, M. Valencia-Pabon, J-C. Vera, On approximating the b-chromaticnumber, Discrete

Appl. Math. 146, 106-110, 2005.

[5]B. Effantin and H. Kheddouci, The b-chromatic number of some power graphs, Discrete Mathematics and Theoretical Computer Science, Vol. 6(1), 45-54, 2003.

[6] B. Effantin and H. Kheddouci, Exact values for the b-chromatic number of a Powercomplete k-ary tree, J. Discrete Math. Sci. Cryptogr., 8, 117-129, 2005.

[7] R.W. Irving, D.F. Manlove, The b-chromatic number of a graph, Discrete Appl.Math. 91, $127-$

$141,1999$.

[8] R. Javadi and B. Omoomi, On b-coloring of the Kneser graphs, DiscreteMathematics,309(13), 4399-4408, 2009.

[9] J. Kratochv'11, Zs. Tuza and M. Voigt, On the b-chromatic number of a graph,Lecture Notes in Comput. Sci. 2573, 310-320, 2002.

[10] S. Shaebani, On the b-chromatic number of regular graphs without 4-cycle, Discrete Applied

Mathematics, Vol. 160,1610-1614, 2012.

[11] J. V., Vivin and M. Vekatachalam,. On b-chromatic number of sun let graph and wheel graph families,Journal of the Egyptian Mathematical Society,23(2), pp. 215-218, 2015. 